\title{
ABBIAMO PUBBLICATO
}

N. 1/2001

Globalizzazione e sviluppo locale di Giacomo Vaciago Labor standard e competitività territoriale di Ilario Favaretto Diversità socio-territoriali e sistemi di welfare di Paolo Calza Bini Istituzioni economiche e economia delle istituzioni nei sistemi produttivi locali di Sebastiano Fadda Verso una lettura integrata dei distretti industriali di Maurizio Mistri Economia globale e cambiamento politico: imprese, istituzioni e società locali alla ricerca di nuove regole di Giancarlo Corò Sviluppo locale e competizione tra territori: Marche e Albania di Roberto Esposti, Franco Sotte, Matteo Taffi

N. 2/2001

La commistione fra economia legale ed economia criminale di Guido M. Rey La sicurezza come componente dello sviluppo territoriale di Vito D'Ambrosio Esclusione urbana e sistemi locali di welfare in Europa di Enzo Mingione $e$ Alberta Andreotti Il locale nella metropoli: riflessioni sul caso di Roma di Fulvio Pellegrini Immigrazione e modello di sviluppo economico italiano di Maurizio Mistri La cooperazione transfrontaliera nell'area adriatica di Gabriele Orcalli

N. 3/2001

Decentramento delle politiche pubbliche e sistemi locali. Alcune riflessioni di base di Sebastiano Fadda L'evoluzione del ruolo dello Stato nell'ordine economico: finanze pubbliche e regolazione economica in Italia, 1970-1997 $d i$ Stefano Solari Nuovi e vecchi strumenti per lo sviluppo locale alla prova dei cambiamenti del quadro istituzionale di Michele Capriati L'evoluzione del distretto: il caso dell'area a sud di Ancona di Giovanni Dini Spagna, una via intermedia allo sviluppo del welfare di Luis Moreno

N. 4/2002

Globalizzazione e processi di governance nei distretti industriali di Maurizio Mistri Globalizzazione e regionalizzazione di Ilario Favaretto Pensare locale e agire globale di Luca Santamaria Divari di sviluppo e politiche regionali: i risultati di alcune simulazioni di Roberto Basile e Marianna Mantuano Il controllo direzionale nei pubblici uffici: l'importanza della funzione statistica di Antonio Cortese Le famiglie valutano i servizi socio-sanitari. Risultati di un'analisi in Italia e nel Triveneto di Mario Bolzan 


\begin{abstract}
N. $\mathbf{5 / 2 0 0 2}$
Competitività, dimensione d'impresa, territorio. Le forze dell'innovazione nel sistema Italia di Ilario Favaretto Economia digitale e trasformazioni del mondo del lavoro di Maurizio Mistri Le politiche per i distretti industriali: da strumento "speciale" a occasione per una nuova politica economica regionale - parte I di Giancarlo Corò Politiche di sviluppo regionale in Italia: obiettivi, strumenti e risultati di Roberto Basile e Marianna Mantuano L'indice di Sviluppo Umano Regionale. Un'applicazione all'Unione Europea di Salvatore Monni
\end{abstract}

N. $6 / 2002$

Ricerca pubblica e universitaria e cluster di PMI in Italia di Andrès RodriguezPose e Maria Cristina Refolo Sviluppo e strategie per l'innovazione di Sergio Ferrari Gli effetti della delocalizzazione internazionale nei Paesi Terzi Mediterranei. Un'analisi per il comparto del "made in Italy" di Roberto Schiattarella Le politiche per i distretti industriali: da strumento "speciale" a occasione per una nuova politica economica regionale - parte II di Giancarlo Corò Le politiche comunitarie per le PMI: recenti tendenze e casi emblematici di Sara Bottoni e Aurelio Bruzzo I sistemi locali del lavoro come mercati locali del lavoro di Marco Rangone

N. $7 / 2003$

Il project financing ed i servizi universali a rilevanza locale di Lorenzo Robotti Trasporto pubblico locale, efficienza e sussidi pubblici: una analisi esplorativa $d i$ Paolo Liberati e Edoardo Marcucci La diversificazione produttiva delle public utilities: alcune esperienze marchigiane di Rosalba Rombaldoni La riforma del TFR: un'analisi critica di Stefano Solari L'innovazione e la tecnologia: quale strategia per le politiche regionali? di Luca Santamaria Il milieu istituzionale, palestra di formazione della cultura locale d'impresa. Considerazioni tratte da un caso di studio del Veneto di Augusto Cucinato

N. $8 / 2003$

Dimensioni di imprese e globalizzazione di Joseph Stiglitz Innovazione e ambiente, fattori di competitività delle economie regionali di Ilario Favaretto Innovazione e nuovi materiali nei sistemi di piccole e medie imprese di Giacomo Moriconi L'innovazione e la tutela ambientale viaggiano insieme di Marta Amadei L'emergere della cooperazione e il caso del "distretto industriale marshalliano" come habitat socio-economico di Maurizio Mistri Le tecnologie di rete nei distretti industriali: un confronto fra Lombardia e Veneto di Maria Chiarvesio, Mario Minoja

N. $9 / 2003$

Politiche di inserimento: la diversità resiste all'integrazione europea di JeanClaude Barbier E-government, innovazione istituzionale e sviluppo locale $d i$ Sebastiano Fadda L'opzione della piccola impresa nello sviluppo economico della ex Rdt dopo la riunificazione tedesca di Michela Parise La difficoltà del 
trasferimento tecnologico e la formazione a distanza promossa dall'ENEA $d i$ Anna Moreno Il lavoro artigiano e l'innovazione tecnologica di Paolo Gurisatti

\section{N. 10/2004}

Politiche urbane e mercato Internazionale: uno studio comparativo di Paul Kantor La "sostenibilità" del progetto urbano, questioni aperte di Vieri Quilici Mercati della tecnologia e strategie tecnologiche. Il caso dell'industria chimica italiana di Fabrizio Cesaroni Il contesto socioeconomico e le occasioni di sviluppo: un'analisi per area problema di Sarah Vanin, M. Bruna Zolin L'autonomia contabile degli enti strumentali regionali: armonia o anarchia? Il caso della Regione Marche di Fabio Giulio Grandis

\section{N. 11/2004}

Finanza e sviluppo economico: il ruolo di Basilea 2 di Giorgio Calcagnini Basilea 2: norme, opportunità e limiti di Alberto Piccoli Il nuovo Accordo di Basilea sul capitale delle banche: le principali caratteristiche e il trattamento dei crediti alle imprese di Francesco Cannata e Sebastiano La viola Politiche degli impieghi e relazioni di clientela: alcune considerazioni sull'impatto della regolamentazione di Basilea 2 sul mercato del credito alle Pmi di Alessandro Berti Politiche settoriali e politica industriale. Il caso italiano di Maurizio Mistri

\section{N. 12/2004}

L'alternativa europea al sogno americano di Jeremy Rifkin Valutazione ex ante delle politiche europee di ICTS: il trade-off tra efficienza e coesione di Roberta Capello e Alessia Spairani Il ruolo della finanza nelle Pmi orientate allo sviluppo di Ilario Favaretto La "lettura" di un sistema locale attraverso l'analisi di un triennio di bilanci ufficiali di Ferruccio Bresolin Competence value e merito finanziario delle imprese di Guido Mantovani

\section{N. 13/2005}

Ricerca, innovazione e ricadute sul sistema produttivo regionale. Il caso delle Marche di Ilario Favaretto e Antonello Zanfei Dopo il declino. Nuovi percorsi di sviluppo per le economie di piccola impresa di Giancarlo Corò Il ciclo virtuoso Conoscenza - Formazione - Apprendimento - Innovazione per l'impresa del nuovo millennio di Sergio Grande e Anna Moreno Specificità finanziarie delle medie imprese italiane: un'analisi del campione Mediobanca-Unioncamere $d i$ Daniela Venanzi "Città metropolitana" e problema della governance. Il caso italiano di Maurizio Mistri

\section{N. 14/2005}

Per una integrazione tra politiche del lavoro e politiche di sviluppo di Sebastiano Fadda Il lavoro atipico in Italia: caratteristiche, diffusione e dinamica di Leonello Tronti e Francesca Ceccato I servizi per l'impiego nelle aree metropolitane. Prime riflessioni di Paolo Calza Bini e Fulvio Pellegrini Distretti industriali e 
agglomerazioni territoriali in Italia. Lo stato delle conoscenze e i problemi di ricerca di Gianfranco Viesti

\section{N. $15 / 2005$}

I distretti industriali come economie della conoscenza di Arnaldo Camuffo e Roberto Grandinetti Internazionalizzazione, innovazione e performance delle PMI dei distretti industriali di Maria Chiarvesio La struttura del costo del lavoro in Italia e nella Unione Europea di Stefania Cardinaleschi Importanza delle infrastrutture a Banda Larga per la diffusione dell'e-Government di Davide Arduini e Sandro Nepi

\section{N. $16 / 2006$}

Il futuro dell'Europa di Jean-Paul Fitoussi La Strategia dell'Occupazione in Europa e in Italia: un approccio istituzionalista di Pasquale Tridico I sistemi informativi geografici (GIS) e le nuove applicazioni dell'informazione georeferenziata di Luciano Stefanini Sistemi informativi geografici come estensione del Customer Relationship Management nelle imprese e nei servizi di Federica Tombari II ruolo dei servizi nel processo di riorganizzazione della produzione nei distretti industriali di Mario Volpe

\section{N. $17 / 2006$}

Competitività e coesione nella nuova programmazione comunitaria di Sebastiano Fadda Competitività contro coesione? Territori e società in Europa alla prova dell'innovazione di Giancarlo Corò Politica di coesione europea: finanziamento, orientamento e governance. Il dibattito politico sulle riforme di Gianluca Spinaci Competitività contro coesione? di Jean Paul Fitoussi Innovazione, finanza e sviluppo: aspetti del dibattito teorico e dell'evidenza empirica di Ferruccio Bresolin

\section{N. 18/2006}

Parchi tecnologici e scientifici e distretti industriali. La lezione mashalliana della Silicon Valley di Maurizio Mistri Il governo della politica dell'innovazione in Europa di Gabriele Orcalli Design come imprenditorialità culturale di Marco Bettiol e Stefano Micelli Le implicazioni per la finanza locale derivanti dalla mobilità degli individui nelle aree urbane e metropolitane: problematiche metodologiche e prospettive di ricerca di Aurelio Bruzzo e Vittorio Ferri Innovazione nelle strategie di distribuzione delle imprese del distretto del Mobile d'Arte di Bassano del Grappa di Gianluca Toschi

\section{N. 19/2007}

Economia e felicità: quali implicazioni per la politica economica di Ferruccio Bresolin La pressione del tempo nelle democrazie consolidate di Vincenzo Memoli Terzo settore e capitale sociale: strategie per un intervento locale di Marco Accorinti Segregazione, polarizzazione sociale e disuguaglianze ad Atene durante gli anni 90: Aspettative teoriche e differenze dei contesti abitativi di Thomas 
Maloutas Trasformazioni socio-economiche e mutamento della città: il caso di Roma di Silvia Lucciarini e Alberto Violante

\section{N. 20/2007}

Mercato del lavoro e territorio di Sebastiano Fadda Enti locali e governo del mercato del lavoro: l'evoluzione normativa di Manuel Marocco I cambiamenti strutturali del Servizio Sanitario Nazionale e i problemi di sostenibilità finanziaria di Maria Rita Materazzi Atteggiamento verso il rischio di innovazione e piccola impresa di Maurizio Mistri Verso una definizione di High Value Manufacturing di Finbarr Livesey

\section{N. 21/2007}

L'economia della cultura: nascita, evoluzione e prospettive di una disciplina in espansione di Carlofilippo Frateschi Economia della cultura e sviluppo locale $d i$ Giancarlo Corò e Riccardo Dalla Torre La spesa delle Amministrazioni pubbliche per le attività culturali nella prima metà del decennio in corso di Aurelio Bruzzo L'economia ed il sostegno democratico in Europa di Vincenzo Memoli

\section{N. 22/2008}

L'evoluzione del rurale. Teoria e politica per lo sviluppo integrato del territorio di Franco Sotte Sviluppo rurale e approccio distrettuale. Analisi e prospettive per un modello di sviluppo economico diversificato di Andrea Arzeni Dai distretti rurali ai parchi agroalimentari di Gianni Moriani Cambiamenti socio-economici e rescaling amministrativo: il caso del Lazio di Alberto Violante L'analisi economica del crimine organizzato: mafia, mercato e sviluppo economico di Anna Bonanno

\section{N. 23/2008}

Imprenditorialità innovativa: quali politiche per il sostegno della crescita economica? di William J. Baumol, Robert E. Litan, Carl J. Schramm e Robert J. Strom Economie in transizione e allargamento dell'UE: i problemi della governance reticolare e dello sviluppo locale di Sebastiano Fadda I fondi strutturali in un'Europa allargata: un trade-off tra efficienza ed equità? $d i$ Claudio Di Berardino Differenti politiche in favore dei cluster: esistono elementi in comune? di Elena Mazzeo Politiche di active ageing in Europa: barriere e opportunità per la loro implementazione in una analisi comparata europea $d i$ Paolo Calza Bini, Silvia Lucciarini, Livia Fay Lucianetti

\section{N. 24/2008}

Programmi statali per la crescita delle imprese innovative negli Stati Uniti. Una tassonomia di Charles $\mathrm{Ou}$ Il nodo della gestione dei flussi migratori. Una difficile governance di Maurizio Mistri Competitività, imprenditorialità e immigrazione. II caso del Veneto di Valentina De Marchi e Eleonora Di Maria Immigrazione e sviluppo locale nel Made in Italy di Eduardo Barberis La popolazione non residente nelle maggiori aree urbane e metropolitane italiane: i risultati di una 
verifica empirica circa le implicazioni per i bilanci dei Comuni coinvolti $d i$ Aurelio Bruzzo, Vittorio Ferri

\section{N. 25/2009}

Come conciliare la solidarietà sociale e l'efficienza economica nell'era della globalizzazione: un punto di vista "regolazionista" di Robert Boyer Le condizioni contrattuali e previdenziali del lavoro degli anziani: politiche e interventi di attuazione di Marco Accorinti e Paolo Calza Bini Anzianità attiva e salute in Europa: il progetto ActivAge di Sandro Turcio e Paolo Calza Bini Mercato del lavoro e settore cooperativo in Italia di Andrea Bernardi e Pasquale Tridico Dall'urbanistica al governo del territorio: i nuovi orientamenti della pianificazione e delle politiche urbane di Lorenzo Ranzato 